biliary complications during long-term follow-up were analyzed. (figure 1)

Results Basic patient characteristics were not significantly different between the groups that underwent EPLBD $(n=164)$ and ES-LBD $(n=52)$. EPLBD compared with ES-LBD resulted in similar outcomes in terms of overall successful stone removal (99.4\% vs $100 \%, \mathrm{P}=0.76)$ and ERCP-related adverse events $(7.9 \%$ vs $5.8 \%, \mathrm{P}=0.77)$. The mean duration of the follow-up was 70.5 months and 65.1 months who underwent EPLBD and ES-LBD, respectively $(\mathrm{P}=0.17)$. A significantly higher incidence of late biliary complications was observed in the ES-LBD group than EPLBD group(12 [23.1\%] vs 18 [11.0\%]; $\mathrm{P}=0.04)$. Multivariate analysis showed that endoscopic sphincterotomy (EST) (OR, 2.407; 95\%CI, 1.0545.501; $\mathrm{P}=0.037)$ and mechanical lithotripsy (ML) (OR, 2.981; 95\%CI, 1.272-6.987; $\mathrm{P}=0.012$ ) were independent risk factors for late biliary complications.

Conclusions During long-term follow-up, patients who underwent ES-LBD had significantly more late biliary complications than those after EP-LBD. EST and ML, special attention should be paid to the possible occurrence of late biliary complications.

\section{IDDF2019-ABS-0148 FOCAL LIVER LESION CLASSIFICATION USING A CONVOLUTIONAL NEURAL NETWORK BASED TRANSFER-LEARNING ALGORITHM ON TRI-PHASE IMAGES OF CONTRAST-ENHANCED ULTRASOUND}

${ }^{1}$ Hang-Tong Hu*, 'Ming Kuang, 'Ming-De Lu, 'Xiao-Yan Xie, ' ${ }^{1}$ Sui Peng, 'Wei Wang, ${ }^{2}$ Xin Li. ${ }^{1}$ The First Affiliated Hospital of Sun Yat-Sen University, China; ${ }^{2}$ Research Center of GE Healthcare, China

\subsection{6/gutjnl-2019-IDDFabstracts.274}

Background To investigate the potential of a deep learning convolutional neural network (CNN) model for focal liver lesion (FLL) differentiation using tri-phase images of contrastenhanced ultrasonography (CEUS) and transfer-learning algorithm. And to preliminarily explore the integration of radiologist experience and artificial intelligence in medical imaging. Methods CEUS images of 527 patients over three phases (unenhanced, early arterial and late portal phases) were manually
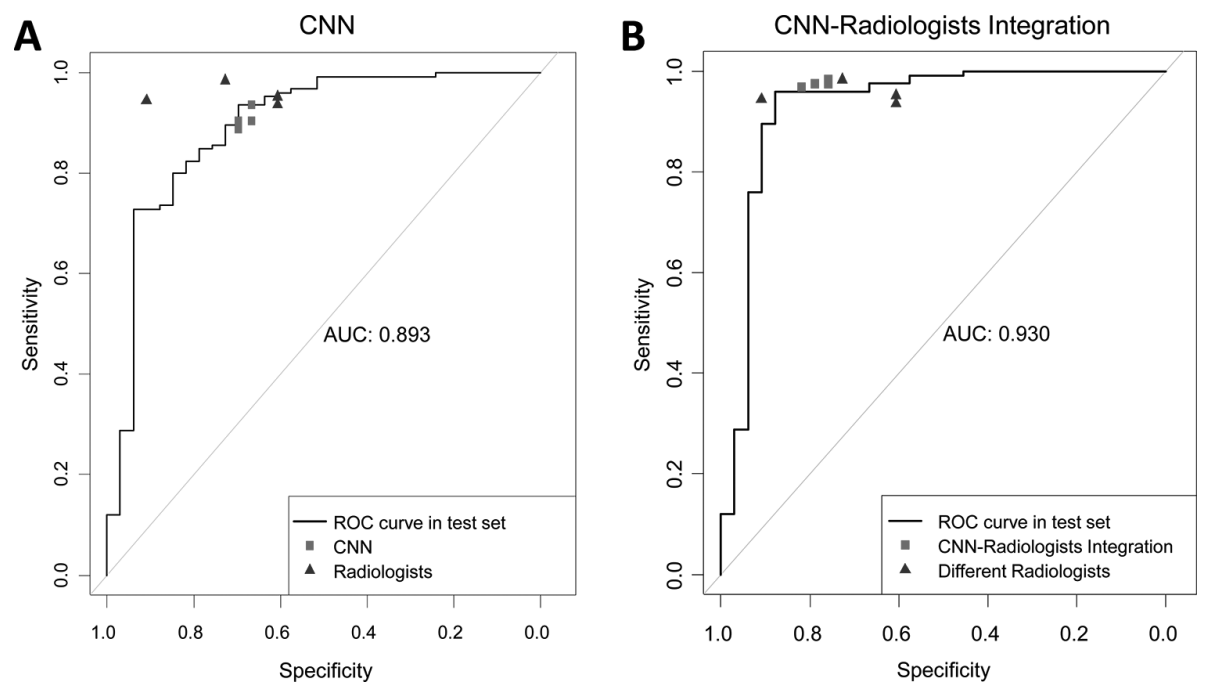

pre-processed into square frames. Supervised CNN was designed as the 152-layer ResNet trained on each phase frames separately and assembled by SVM algorithm. The model was trained on 55350 augmented frames and tested on 474 un-augmented frames. The performance was evaluated on stability and efficiency, and compared with radiologist diagnoses which were based on pre-processed frames first and then on videos with full access to patients' clinical information. Integration of $\mathrm{CNN}$ and radiologist experience was performed through assemble of the CNN outputs and radiologist diagnoses by SVM algorithm.

Results The CNN model was stable among radiologists (ACC: 0.85-0.88; SEN: 0.89-0.94; SPE: 0.67-0.70). While radiologist diagnosed basing on frames varied significantly (ACC: $0.65-$ 0.82; SEN: 0.62-0.90; SPE: 0.55-0.82) and no rules could be found between the variation and radiologists' experience. However, for diagnoses based on videos, the trend could be found that SPE increased with a higher radiologist's experience $(0.61$ vs. $0.91, P<0.01)$. For diagnostic efficiency, the frames based $\mathrm{CNN}$ model and videos based diagnoses were similar for the less-experienced radiologist. While for the experienced radiologist, the CNN model had a significantly lower SPE (0.70 vs. $0.91, P<0.01$ ) (figure $1 \mathrm{~A}$ ). Integration of the $\mathrm{CNN}$ and radiologist's experience achieved good stability. Performance was an improvement for the less-experienced radiologist (SPE: 0.76 vs. $0.61, P<0.01)$ but a setback for the experienced radiologist (SPE: 0.79 vs. $0.91, P<0.01$ ) (figure $1 \mathrm{~B}$ ).

Conclusions The CNN model showed good stability that overcame the operator-dependent disadvantage of CEUS. The integrated model of the CNN and radiologist's experience could significantly improve the performance of less-experienced radiologists, but not for the experienced radiologists.

\section{IDDF2019-ABS-0149 ERCP IN THE PATIENTS WITH SITUS INVERSUS TOTALIS: A CASE SERIES}

Wenying Yang*. Digestive Endoscopy Center, Department of Gastroenterology, Ruijin Hospital, Shanghai Jiaotong University School of Medicine, China

\subsection{6/gutjnl-2019-IDDFabstracts.275}

Background Situs inversus totalis (SIT) is a rare congenital disease with complete transposition of all viscera, which makes 


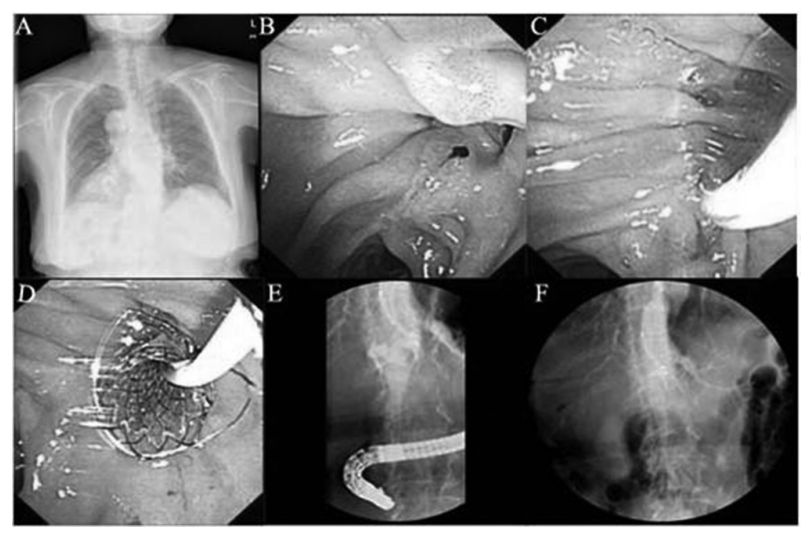

Abstract IDDF2019-ABS-0149 Figure 1

endoscopic procedures, especially endoscopic retrograde cholangiopancreatography (ERCP) complicated and challenging. The aim of this study was to report and discuss the patients with SIT underwent ERCP in our center.

Methods A retrospective review of the databases was performed, and case series of patients with SIT underwent ERCP was reported. (figure 1)

Results Nine ERCP procedures were performed in 4 patients: 2 males and 2 females, 3 patients were in their fifties, and 1 patient was in her eighties. The diagnosis after ERCP included: benign or malignant biliary stricture and bile duct stones. Based on the individual situation, endoscopic retrograde biliary drainage, stone extraction, endoscopic naso-biliary drainage, endoscopic metal biliary endoprosthesis was performed. All procedures were performed successfully, except for in one patient, whose biliary stricture was very serious, hindering the procedure. No ERCP-related adverse events were reported.

Conclusions ERCP can be safely and effectively performed in patients with SIT.

\section{IDDF2019-ABS-0167 IDENTIFICATION OF CANCER-RELATED EXOSOMAL MIRNAS IN NASH-INDUCED LIVER CIRRHOSIS WITH HEPATOCELLULAR CARCINOMA}

${ }^{1}$ Allia Najmie Muhammad Yusuf*, ${ }^{2}$ Raja Affendi Raja Ali, ${ }^{2}$ Khairul Najmi Muhammad Nawawi, 'Norfilza Mohd Mokhtar. 'Department of Physiology, Faculty of Medicine, Universiti Kebangsaan Malaysia, Kuala Lumpur, Malaysia; ${ }^{2}$ GUT research group, Gastroenterology Unit, Department of Medicine, UKM Medical Centre, Kuala Lumpur, Malaysia

\subsection{6/gutjnl-2019-IDDFabstracts.276}

Background The objective of this study was to identify the presence of selected miRNAs in exosomes of the serum and ascitic fluid and to determine their expression levels in patients with NASH-related liver cirrhosis with or without HCC

Methods A literature search was performed to identify potential miRNAs involved in the pathogenesis of HCC. The expression of the miRNA candidates in the exosome of serum and ascitic fluid were investigated in 52 patients of NASH-induced liver cirrhosis with HCC $(n=26)$ as compared to NASH-induced liver cirrhosis without HCC $(n=26)$ by using quantitative realtime PCR. Fold change was calculated using $2^{\wedge-{ }^{-C C T}}$ method. Results The median age for patients with NASH-induced liver cirrhosis with HCC was 71.5 years old versus the control group of 60.5 years old. More than 50\% of the patients in both groups had underlying comorbidities such as diabetes mellitus, hypertension, and dyslipidemia. Three oncogenic miRNAs (miR182, miR-301a, and miR-373) with high expression in the HCC tissues were selected as the candidate miRNAs. The expression level of serum exosomal mir-182, miR-301a, and miR-373 were significantly upregulated with fold change (fc) of $1.77,2.52$, and $1.67(\mathrm{p}<0.05)$ respectively in NASH-induced liver cirrhosis with HCC as compared to the control group. The same trend could be observed in the expression level of ascitic fluid exosomal mir-182, miR-301a, and miR-373 whereby they were significantly upregulated with fc of 1.6, 1.94 and 2.13 (p <0.05) respectively in NASH-induced liver cirrhosis with $\mathrm{HCC}$ as compared to the control group. However, poor correlation of miRNA expression (miR-182, miR-301a and miR-373) between serum exosomal and ascitic fluid exosomal in HCC group with miR-182 ( $\mathrm{r}=0.213, \mathrm{p}=0.444), \operatorname{miR}-301 \mathrm{a} \quad(\mathrm{r}=-0.242, \mathrm{p}=0.422)$ and miR-373 ( $r=0.24, p=0.427)$.

Conclusions A significant increase in the expression levels of exosomal miR-182, miR-301a, and miR-373 for both serum and ascitic fluid may be potential biomarkers for NASHinduced liver cirrhosis with HCC

\section{IDDF2019-ABS-0168 BONE AND RENAL SAFETY ARE IMPROVED IN CHRONIC HBV PATIENTS 1 YEAR AFTER SWITCHING TO TENOFOVIR ALAFENAMIDE (TAF) FROM TENOFOVIR DISOPROXIL FUMARATE (TDF)}

${ }^{1}$ Henry Lik Yuen Chan*, ${ }^{2}$ Wai Kay Seto, ${ }^{3}$ Maria Buti, ${ }^{4}$ Namiki Izumi, ${ }^{5}$ Young-Suk Lim, ${ }^{6}$ JiaHorng Kao, ${ }^{7}$ Adrian Streinu-Cercel, ${ }^{8}$ Elena Nurmukhametova, ${ }^{9}$ Xiaoli Ma, ${ }^{10}$ Fehmi Tabak, ${ }^{11}$ Maciej Jablkowski, ${ }^{12}$ Vithika Suri, ${ }^{12}$ John Flaherty, ${ }^{12}$ Audrey Lau, ${ }^{12}$ Anuj Gaggar, ${ }^{12}$ Shuyuan Mo, ${ }^{13}$ Abhijit Chowdhury, ${ }^{14}$ Scott Fung, ${ }^{15}$ Wan-Long Chuang, ${ }^{16}$ Edward Gane. ${ }^{1}$ Institute of Digestive Disease, State Key Laboratory of Digestive Disease, The Chinese University of Hong Kong, Hong Kong; ${ }^{2}$ Queen Mary Hospital, Hong Kong; ${ }^{3}$ Vall d'Hebron Barcelona Campus Hospitalari, Barcelona, Spain; ${ }^{4}$ Japanese Red Cross Musashino Hospital, Tokyo, Japan; ${ }^{5}$ Asan Medical Center, University of Ulsan College of Medicine, Seoul, Korea, South; ${ }^{6}$ Graduate Institute of Clinical Medicine, National Taiwan University Hospital, Taipei, Taiwan; 'Institutul National de Boli Infectioase 'Prof.Dr. Matei Bals,' Bucharest, Romania; ${ }^{8}$ Infectious Clinical Hospital \#1 of Moscow Healthcare Department, Russia; ${ }^{9} P C$, Bryn Mawr, $P A$, USA; ${ }^{10}$ Istanbul Üniversitesi Cerrahpaşa Tip Fakültesi Hastanesi, Istanbul, Turkey; ${ }^{11}$ Oddzial Obserwacyjno-Zakazny, Poland; ${ }^{12}$ Gilead Sciences, Inc., Foster City, CA, USA; ${ }^{13}$ School of Digestive and Liver Diseases, Institute of Post Graduate Medical Education and Research, Kolkata, India; ${ }^{14}$ University of Toronto, Ontario, Canada; ${ }^{15}$ Kaohsiung Medical University Chung-Ho Memorial Hospital, Kaohsiung, Taiwan; ${ }^{16}$ Auckland Clinical Studies, Auckland, New Zealand

\subsection{6/gutjn|-2019-IDDFabstracts.277}

Background TAF has shown efficacy non-inferior to that of TDF at Week 96 with less bone and renal effects. Following implementation of a protocol amendment to extend double-blind (DB) treatment for an additional year, 50\% of patients were able to continue on DB treatment while the remainder had already rolled-over to open-label (OL) TAF at Week 96. Here we compared the efficacy and safety from Week 96 to 144 in patients randomized to TDF in whom treatment was either continued or switched to TAF at Week 96.

Methods In 2 identically-designed studies, 1298 HBeAg-negative and HBeAg-positive $\mathrm{CHB}$ patients (873 TAF, $425 \mathrm{TDF}$ ) were randomized and treated. In the TDF group, 211 remained on TDF (DB TDF) while 180 patients were switched to OL TAF (TDF $\rightarrow$ TAF) at Week 96. Safety assessments including changes in bone (hip and spine BMD) and 